\title{
Progress in defining autonomic consequences of seizure activity including sudden death
}

\author{
Mark Stewart ${ }^{1}$
}

Received: 31 December 2018 / Accepted: 9 January 2019 / Published online: 25 January 2019

๑) Springer-Verlag GmbH Germany, part of Springer Nature 2019

Keywords Epilepsy · Seizures $\cdot$ SUDEP $\cdot$ Autonomic $\cdot$ Arrhythmia

Sudden unexpected death in epilepsy (SUDEP) is the leading cause of epilepsy-related deaths (e.g., [1]), and has drawn continuously increasing interest from basic and clinical investigators as evidenced by publications [2] and committed research funding. There has been an exponential increase in the number of review articles (40 in total) focusing on SUDEP over the last 20 years (Fig. 1).

The review by Barot and Nei in this issue of Clinical Autonomic Research [3] is significant because it (1) is accessible (6 pages of text and 102 references), (2) clearly summarizes clinical experience, which helps to form translational targets, (3) summarizes current thinking from a clinical perspective, which is important for moderating the penetration of preclinical data into clinical thinking, and (4) helps with convergence on standard terminology. Its focus is on autonomic aspects of SUDEP, touching on the main concepts: basic epidemiology, the MORTality in Epilepsy Monitoring Unit Study (MORTEMUS), ictal and interictal cardiac arrhythmias, other cardiovascular metrics, respiratory derangements (including laryngospasm), and approaches for SUDEP prevention.

Obvious triggers to update or revisit a review of a topic such as SUDEP are major changes in the clinical definitions [4], large studies that establish critical aspects of the clinical picture [5], and new perspectives on mechanism. The timing and opening content of most reviews suggest their individual trigger(s).

The review by Bardot and Nei serves the additional function of introducing seizure-related pathophysiology

Mark Stewart

mark.stewart@downstate.edu

1 Departments of Physiology \& Pharmacology, and Neurology, State University of New York Downstate Medical Center, 450 Clarkson Avenue, Brooklyn, NY 11203, USA to a broader clinical autonomic community. The autonomic issues that occur during seizure activity, persist after repeated seizures, and permit brain activity to be lethal are incredibly interesting (to many of us), but there are lessons to be learned in separating those autonomic issues that may be responsible for lasting or lethal cardiorespiratory dysfunction from those autonomic phenomena that are intended to safeguard an individual's survival.

For extreme brain activity to be lethal, the autonomic nervous system must be perverted or overwhelmed. The key concepts are laid out in the Barot and Nei review and the interested reader can find milestones in the cited literature that certainly contributed to increased interest in autonomic consequences of seizure activity and the mechanisms of sudden death in epilepsy.

Our own research has followed a path that resembles the authors' approach in their review: What is known to happen in epilepsy patients? What are the worst-case cardiac consequences of a seizure? What are the worst-case respiratory consequences of a seizure? What are the long-term, and possibly cumulative, consequences of repeated seizures? Do any of these consequences appear useful for stratifying risk for sudden death or can these consequences be exploited in other preventative ways?

We introduced our main data set on laryngospasm-mediated obstructive apnea as the cause of SUDEP in a rat model in 2016 [6] and a description of biomarkers directly linking our preclinical data to the data from MORTEMUS [7, 8]. Importantly, laryngospasm with partial airway closure was a common occurrence, but occasionally, an extreme event (complete airway closure from laryngospasm) occurred, producing obstructive apnea, which could lead to an extreme consequence (respiratory arrest and eventual death). Along the way, we have been amazed at the capabilities of the autonomic nervous system as we sought to define its limits in different contexts. 


\section{PUBMED listed SUDEP reviews 1999 - present}

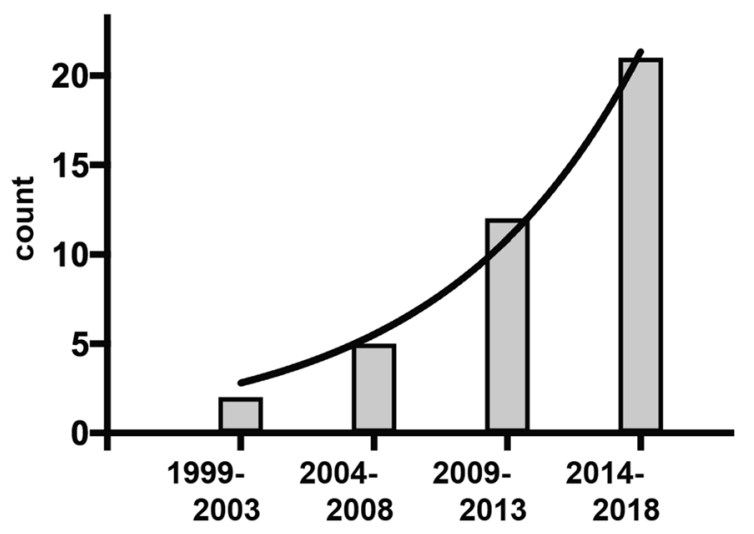

Fig. 1 Exponential increase in published review articles on sudden unexpected death in epilepsy (SUDEP) over the last 20 years (1999present). Counts are binned in 5-year intervals. PubMed search criterion: "SUDEP [Title] AND review [Publication Type]". $R^{2}$ for the exponential regression line is 0.989 . Doubling time is every 5 years

Normal autonomic responses to extreme events such as airway closure certainly add to or interact with autonomic activity driven by the seizure itself. The dissection of autonomic, cardiovascular, and respiratory activity that may be a "normal" response to an extreme event from activity directly driven by an epileptic seizure is where animal models can contribute greatly. It is critical to understand autonomic reactions to these extreme conditions to be able to identify what physiological systems and conditions might actually be abnormal at the outset and thus legitimately identified as a feature of an individual that could predict risk.

Reviews such as the one by Barot and Nei offer context, and that context engages investigators-such as myselfseeking this valuable information for future experiments; and clinical faculty and trainees who strive to remain current with the state-of-the-art, in spite of the ever-increasing stream of information.
Funding None.

\section{Compliance with ethical standards}

Conflict of interest All authors declare that they have no conflict of interest.

\section{References}

1. Devinsky O, Spruill T, Thurman D, Friedman D (2016) Recognizing and preventing epilepsy-related mortality: a call for action. Neurology 86:779-786

2. Scorza FA, Carmo AC, Scorza CA, Fiorini AC (2017) SUDEP: a steep increase in publication since its definition. Epilepsy Behav 72:195-197

3. Barot N, Nei M (2018) Autonomic aspects of sudden unexpected death in epilepsy (SUDEP). Clin Auton Res. https://doi. org/10.1007/s10286-018-0576-1

4. Nashef L, So EL, Ryvlin P, Tomson T (2012) Unifying the definitions of sudden unexpected death in epilepsy. Epilepsia 53:227-233

5. Ryvlin P, Nashef L, Lhatoo SD, Bateman LM, Bird J, Bleasel A, Boon P, Crespel A, Dworetzky BA, Hogenhaven H, Lerche H, Maillard L, Malter MP, Marchal C, Murthy JM, Nitsche M, Pataraia E, Rabben T, Rheims S, Sadzot B, Schulze-Bonhage A, Seyal M, So EL, Spitz M, Szucs A, Tan M, Tao JX, Tomson T (2013) Incidence and mechanisms of cardiorespiratory arrests in epilepsy monitoring units (MORTEMUS): a retrospective study. Lancet Neurol 12:966-977

6. Nakase K, Kollmar R, Lazar J, Arjomandi H, Sundaram K, Silverman J, Orman R, Weedon J, Stefanov D, Savoca E, Tordjman L, Stiles K, Ihsan M, Nunez A, Guzman L, Stewart M (2016) Laryngospasm, central and obstructive apnea during seizures: defining pathophysiology for sudden death in a rat model. Epilepsy Res 128:126-139

7. Lacuey N, Vilella L, Hampson JP, Sahadevan J, Lhatoo SD (2018) Ictal laryngospasm monitored by video-EEG and polygraphy: a potential SUDEP mechanism. Epileptic Disord 20:146-150

8. Stewart M, Kollmar R, Nakase K, Silverman J, Sundaram K, Orman R, Lazar J (2017) Obstructive apnea due to laryngospasm links ictal to postictal events in SUDEP cases and offers practical biomarkers for review of past cases and prevention of new ones. Epilepsia 58:e87-e90 